Rapid Reviews COVID-19

\title{
Review 3: "Antisense \\ oligonucleotides target a \\ nearly invariant structural \\ element from the SARS- \\ CoV-2 genome and drive \\ RNA degradation"
}

Valerio Orlando ${ }^{1}$

${ }^{1}$ KAUST, BESE, Saudi Arabia

Published on: Nov 05, 2020

DOI: $10.1162 / 2 \mathrm{e} 3983 f 5 . e 3 f 045 b 2$

License: Creative Commons Attribution 4.0 International License (CC-BY 4.0). 


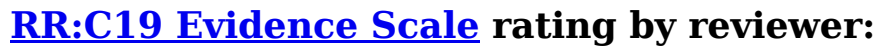

- Potentially informative. The main claims made are not strongly justified by the methods and data, but may yield some insight. The results and conclusions of the study may resemble those from the hypothetical ideal study, but there is substantial room for doubt. Decision-makers should consider this evidence only with a thorough understanding of its weaknesses, alongside other evidence and theory. Decisionmakers should not consider this actionable, unless the weaknesses are clearly understood and there is other theory and evidence to further support it.

******************************************

\section{Review:}

The work by Lulla et al. describes an AntiSense-Oligo (ASO) approach to target COVID19 RNA. The rationale is to use LNA ASO to target specific portions of the viral genome to inhibit its replication.

LNA technology is well known and its use has become a promising strategy in clinics. Advantage is mainly the long term stability of LNA oligos as they are not degraded by endogenous RNAi machinery.

By computational approach authors identify a critical, conserved portion of the 5'end of SARS-CoV-2 genome. In vitro assays (SHAPE) demonstrate the effectiveness of specific set of the designed LNAs in targeting predicted single strand RNA regions (s2m) of the viral genome.

Using a tissue-culture based assay, the authors convincingly show the ability of s2mLNA to target and degrade a gene expression reporter construct.

Authors complete their analysis by moving to an Astrovirus replication in culture system. Data show ability of selected gap-mers to inhibit viral replication in the nM linear range $(0,5-500)$.

Overall, within the limits of the assays used, the results are consistent with the rationale of the hypothesized knock down strategy. As indicated in the discussion, the work represents a promising preliminary set of data towards preclinical large-scale screening of in vivo effective gap-mers to be used in real pathological contexts. 